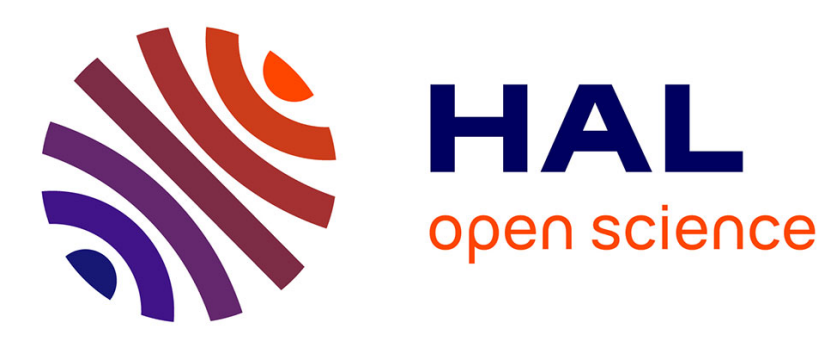

\title{
Motion detection: Fast and robust algorithms for embedded systems
}

Lionel Lacassagne, Antoine Manzanera, Antoine Dupret

\section{To cite this version:}

Lionel Lacassagne, Antoine Manzanera, Antoine Dupret. Motion detection: Fast and robust algorithms for embedded systems. International Conference on Image Processing (ICIP), Nov 2009, Le Caire, Egypt. 10.1109/ICIP.2009.5413946 . hal-01130889

\section{HAL Id: hal-01130889 \\ https://hal.science/hal-01130889}

Submitted on 12 Mar 2015

HAL is a multi-disciplinary open access archive for the deposit and dissemination of scientific research documents, whether they are published or not. The documents may come from teaching and research institutions in France or abroad, or from public or private research centers.
L'archive ouverte pluridisciplinaire $\mathbf{H A L}$, est destinée au dépôt et à la diffusion de documents scientifiques de niveau recherche, publiés ou non, émanant des établissements d'enseignement et de recherche français ou étrangers, des laboratoires publics ou privés. 


\section{MOTION DETECTION: FAST AND ROBUST ALGORITHMS FOR EMBEDDED SYSTEMS}

\author{
L. Lacassagne \\ IEF/AXIS - Digiteo Labs - Université Paris Sud
}

\author{
A. Manzanera \\ UEI - ENSTA - Paris Tech
}

\begin{abstract}
This article introduces a new hierarchical version of a set of motion detection algorithms called $\Sigma \Delta$. These new algorithms are designed to preserve as much as possible the computational efficiency of the basical $\Sigma \Delta$ estimation, in order to target real-time implementation for low power consumption processors and embedded systems.
\end{abstract}

Index Terms - Motion detection, Sigma-Delta filtering, Embedded systems, Real-Time implementation.

\section{INTRODUCTION}

The growing interest for developing fully automatic video surveillance systems has recently renewed the interest for fast and reliable motion detection algorithms. Such algorithms must partition the pixels of every frame of the image sequence into two classes: the background, corresponding to pixels belonging to the static scene (label: 0 ), and the foreground, corresponding to pixels belonging to a moving object (label: 1).

A motion detection algorithm must discriminate the moving objects from the background as accurately as possible, without being too sensitive to the sizes and velocities of the objects, or to the changing conditions of the static scene. For long autonomy and discretion purposes, the system must not consume too much computational resources (energy and circuit area) [1]. As it involves a great amount of data like any image processing module - the motion detection is certainly the most computationally demanding function of a video surveillance system.

Background subtraction techniques have been the object of much attention for years [2]. Recently, we have proposed a new type of methods based on $\Sigma \Delta$ estimation [3]. These methods are very attractive from a computational point of view since they work on any size fixed-point arithmetic using only comparison, increment and absolute difference, while being as robust as other mono-modal statistical estimation methods (e.g. Gaussian estimation), whose computation is much more costly.

Different modified versions of the basical $\Sigma \Delta$ algorithm have been proposed since then. The purpose of this paper is to review and compare them and also to introduce a new hierarchical version.

\section{2. $\Sigma \Delta$ BACKGROUND SUBTRACTION}

The basic principle of the $\Sigma \Delta$ algorithm is to estimate parameters of the background using $\Sigma \Delta$ modulation, which is a very common tool in analog-to-digital conversion: Considering a time-varying signal $f_{t}$ (continuous or discrete), we estimate a discrete signal $d_{t}$ by quantizing the time indexes $\left\{t_{i}\right\}_{i \in \mathbb{N}}$, and then performing at every time index $i$ the following update formulas:

If $d_{t_{i-1}}<f_{t_{i}}$ then $d_{t_{i}}=d_{t_{i-1}}-\varepsilon$ else $d_{t_{i}}=d_{t_{i-1}}+\varepsilon$ where $\varepsilon$ is the discretization step (least significant bit) of $d_{t}$.

In $\Sigma \Delta$ background subtraction, the input signal is the value of every pixel over time $I_{t}$, from which we compute the first $\Sigma \Delta$ background estimator $M_{t}$. Then the values of the absolute differences $\left|I_{t}-M_{t}\right|$ are used to compute the second $\Sigma \Delta$ background estimator $V_{t}$, which is a parameter of dispersion.

\subsection{Basical $\Sigma \Delta$ algorithm}

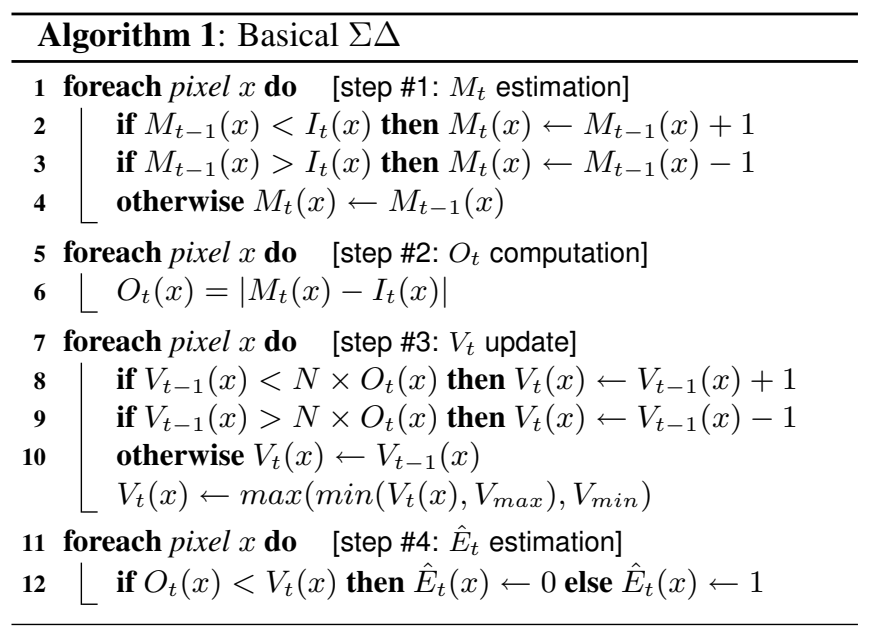

In the basical version (Alg. 1), the $\Sigma \Delta$ background $M_{t}$ and $\Sigma \Delta$ variance $V_{t}$ are updated every frame, according to the comparison with the current image $I_{t}$ and current absolute difference $O_{t}$ respectively. $N$ is an amplification factor for $V_{t}$, allowing then to compute the motion label $\hat{E}_{t}$ by simply comparing $O_{t}$ and $V_{t}$ (typical values of $N$ are between 1 and 4). $V_{\min }$ and $V_{\max }$ are two parameters used to control the 
overflow of $V_{t}$ that could happens if some pixels are saturated (due to sensor over-exposition). Their typical values are 2 and $2^{m}-1$ respectively (where $m$ is the number of bits of the representation). Note that this clipping is a modification not present in the original version [3].

\subsection{Improved algorithm: conditional $\Sigma \Delta$}

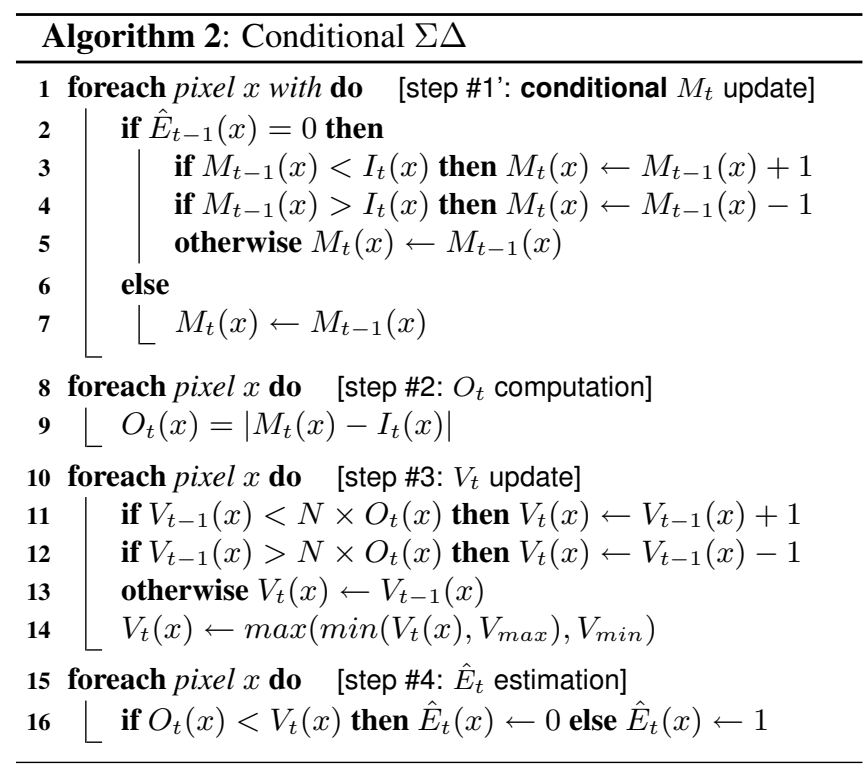

The conditional version (Alg. 2 and Fig. 1) uses relevance feedback from the estimated position of the moving objects at the previous frame, given by $\hat{E}_{t-1}$. It consists in updating the $\Sigma \Delta$ background $M_{t}$ and/or the variance $V_{t}$ only for the pixels $x$ considered background (i.e. where $\hat{E}_{t-1}(x)=0$ ). It prevents moving object from integrating the background and/or modifying the noise variance [4].

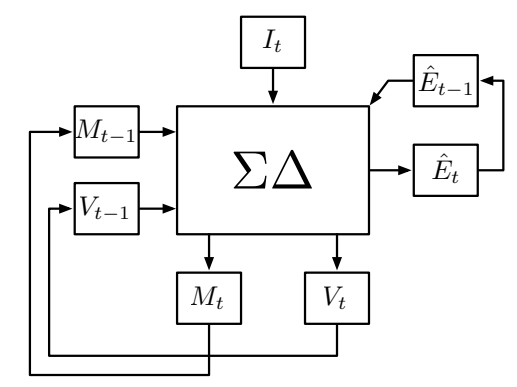

Fig. 1. conditional $\Sigma \Delta$

\subsection{Zipfian estimation}

The Zipfian version (Alg. 3) [5] is based on the relation between the $\Sigma \Delta$ estimation and the statistical estimation, using a Zipf-Mandelbrot distribution, which implies that the updating frequency of the background should be proportional to the dispersion of the distribution (variance). In that version, we first compute a threshold which varies according to the frame index $t: \rho$ is the value of the index modulo $2^{m}(m$ is the number of bits of the representation). $\pi$ is the value of the greatest power of 2 which divides $\rho$. Finally the threshold $\sigma$ is equal to $2^{m}$ divided by $\pi$. The result is that pixels $x$ such that $V_{t}(x)>2^{m-k}$ will be updated every $2^{k-1}$ frames, for $k \in\{1, m\}$. To avoid auto-reference, the variance $V_{t}$ is updated using a constant period $T_{V}$ (usually a power of 2 between 1 and 64). $T_{V}$, like the amplification parameter $N$, can be automatically adjusted using a simple noise estimation method, which consists in counting the number of isolated pixels in the estimated labels $\hat{E}_{t}$.

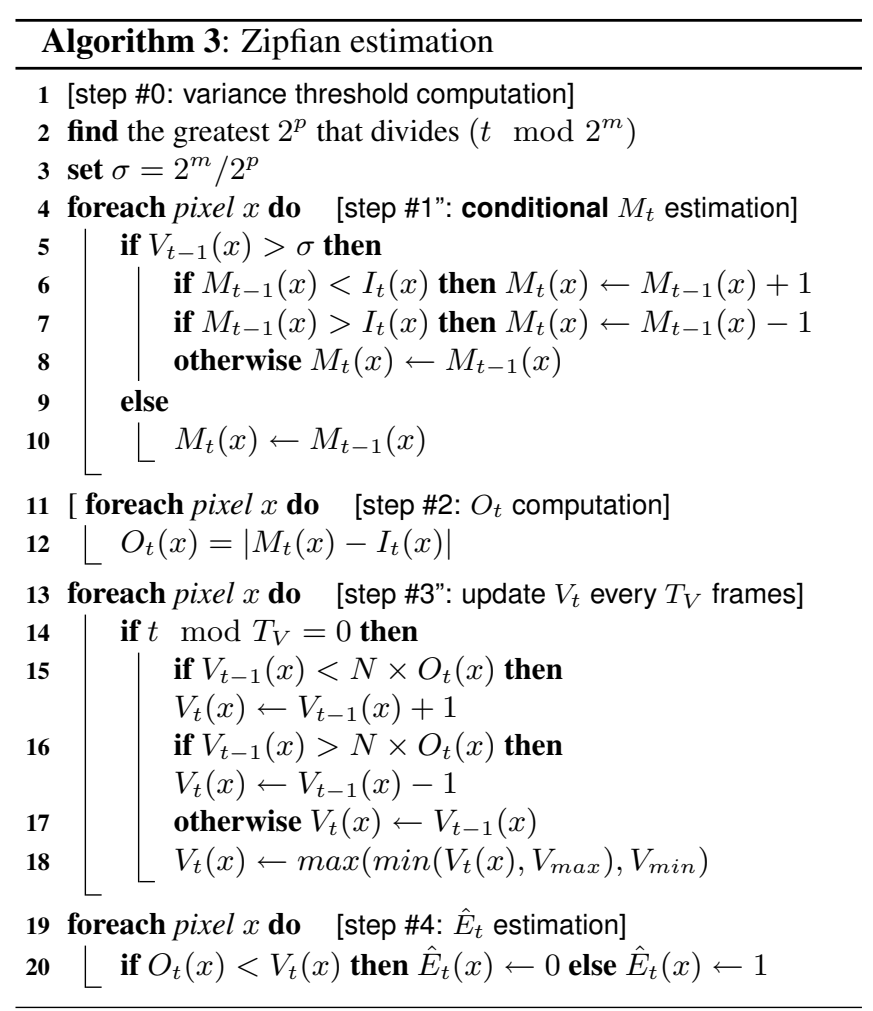

\subsection{New hierarchical algorithm}

The hierarchical algorithm (Fig. 2) is a bi-level version of $\Sigma \Delta$ filtering. Each $\Sigma \Delta$ block implements the basic algorithm \#1 of the algorithm \#3. Both blocks are using conditional update. At the low level it is a conditional temporal update: $M_{t}^{1}$ and $V_{t}^{1}$ are updated depending on $\hat{E}_{t-1}^{1}$. At the high level, it is a conditional spatial update: $M_{t}^{0}$ and $V_{t}^{0}$ are updated depending on $\tilde{E}_{t}^{0}$, the oversampling binary mask of $\hat{E}_{t}^{1}$. The subsampling factor is in the range $[2,10]$ and is set accordingly to the "size" of the clutter noise. Finally, a morphological post-processing is applied in two steps. The first one 


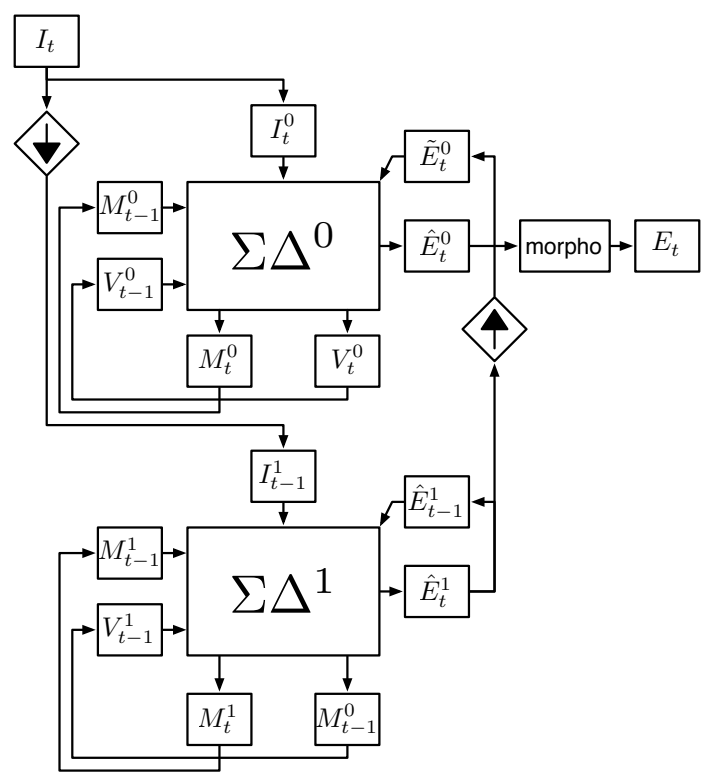

Fig. 2. hierarchic $\Sigma \Delta$

removes stand-alone pixels that are considered as noise, the second one is a $3 \times 3$ morphological closing.

\section{BENCHMARK}
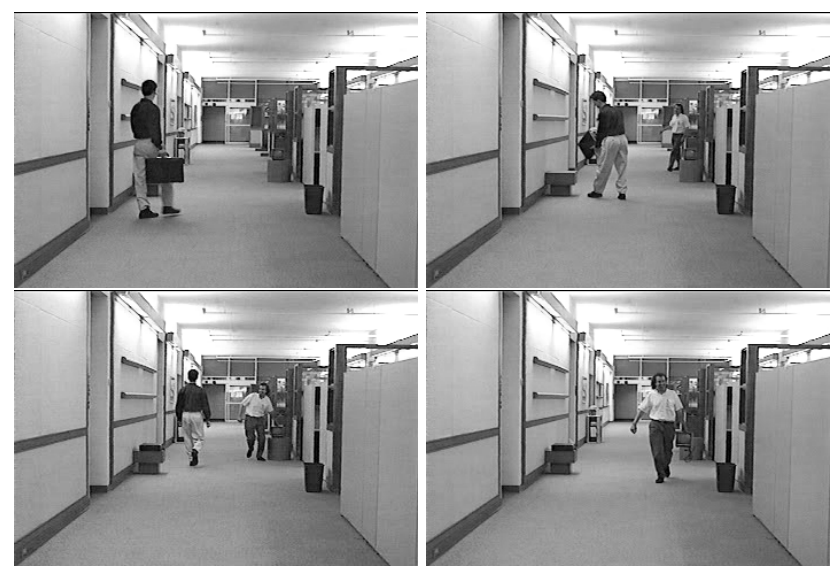

Fig. 3. Hall sequence, images 38, 91, 170, 251

In order to evaluate the impact of the modifications on the performance of these algorithms, a RoC analysis has been done with the Hall sequence (Fig. 3) than can be considered as a difficult sequence because of the radial movement of nonrigid objects (people). The Ground Truth has been drawn for 4 images of that sequence. Given $T P$ the True Positive, $T N$ the True Negative, $F P$ the False Positive and $F N$ the False Negative, we compute the Matthews Correlation Coefficient
(Eq. 1) instead of accuracy or product of $T P$ ratio by $T N$ ratio because the two classes (motion and background) are of very different size. It returns a value between -1 (perfect inverse segmentation) and +1 (perfect segmentation) while 0 signifies a wrong segmentation.

$$
M C C=\frac{T P \times T N-F P \times F N}{\sqrt{(T P+F P)(T P+F N)(T N+F P)(T N+F N)}}
$$

A set of 32 algorithms (combinations of parameters) has been evaluated. Figures (Tab. 1) are provided for only four of them: $\Sigma \Delta$ is the basic algorithm (Fig. 4), $\Sigma \Delta+\operatorname{Zipf}$ (Fig. 5 ) is the basic algorithm with Zipfian estimation, Conditional $\Sigma \Delta$ (Fig. 6) is the best mono-level algorithm with conditional update (with or without Zipfian estimation) and Hierarchical $\Sigma \Delta$ (Fig. 7)is the best two-level algorithm with conditional update. For this benchmark, the decimation factor for subsampling and oversampling was set to 8 and the Zipfian $V_{t}$ update period $T_{V}$ was set to 4 .

\begin{tabular}{|c|c|c|c|c|c|}
\hline algorithm & 38 & 91 & 170 & 251 & average \\
\hline \hline \multicolumn{7}{|c|}{ MCC without morphological post processing } \\
\hline$\Sigma \Delta$ & 0.495 & 0.347 & 0.169 & 0.282 & 0.323 \\
\hline$\Sigma \Delta+$ Zipf & 0.676 & 0.600 & 0.366 & 0.308 & 0.487 \\
\hline Conditional $\Sigma \Delta$ & 0.424 & 0.533 & 0.555 & 0.590 & 0.526 \\
\hline Hierarchical $\Sigma \Delta$ & 0.644 & 0.663 & 0.468 & 0.415 & 0.548 \\
\hline \multicolumn{7}{|c|}{$M C$ with morphological post processing } \\
\hline \multicolumn{7}{|c|}{0.811} & 0.657 & 0.372 & 0.596 & 0.609 \\
\hline Conditional $\Sigma \Delta$ & 0.754 & 0.764 & 0.530 & 0.385 & 0.608 \\
\hline Hierarchical $\Sigma \Delta$ & 0.816 & 0.827 & 0.686 & 0.582 & 0.728 \\
\hline
\end{tabular}

Table 1. Results: $M C C$ scores for $4 \Sigma \Delta$ algorithms with/without morphological post processing

Considering first, the results without post morphological processing, each evolution has better results than the previous one. The best conditional version is obtained with Zipfian estimation combined with the conditional update of $M_{t}$ and $V_{t}$. The best hierarchical version is obtained with the best conditional version combined with a conditional update of $M_{t}^{1}$ at low level. Considering then the results with morphological post processing, all results are in progression except for image \# 170 that corresponds to radial movement of the first person. Both visual and numerical results enforce the use of morphological post-processing (Fig. 8) to remove remaining noise. Another benchmark, not presented here, has been done on a sequence with cars. The results were better but harder to differentiate, as such a kind of sequence is easier to segment.

\section{CONCLUSION}

We have presented a new hierarchical and conditional motion detection algorithm based on an evolution of previous $\Sigma \Delta$ algorithms. Preliminary results show better (visual and 


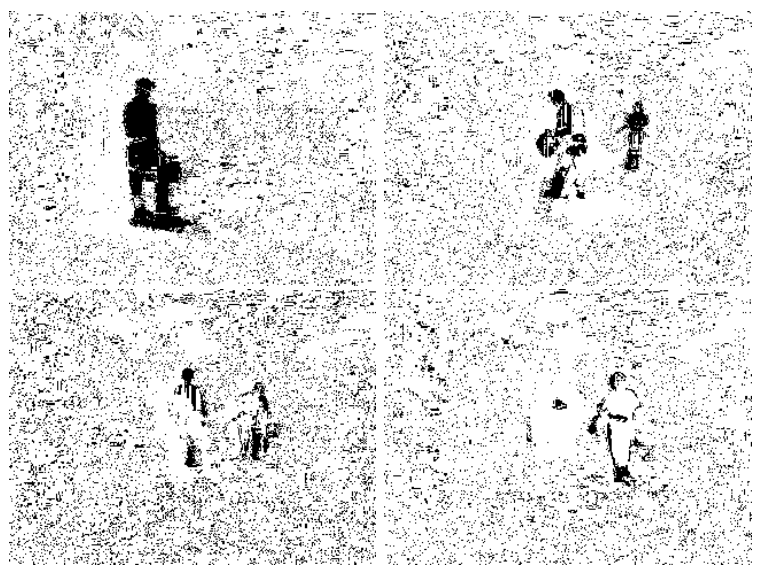

Fig. 4. basic $\Sigma \Delta$

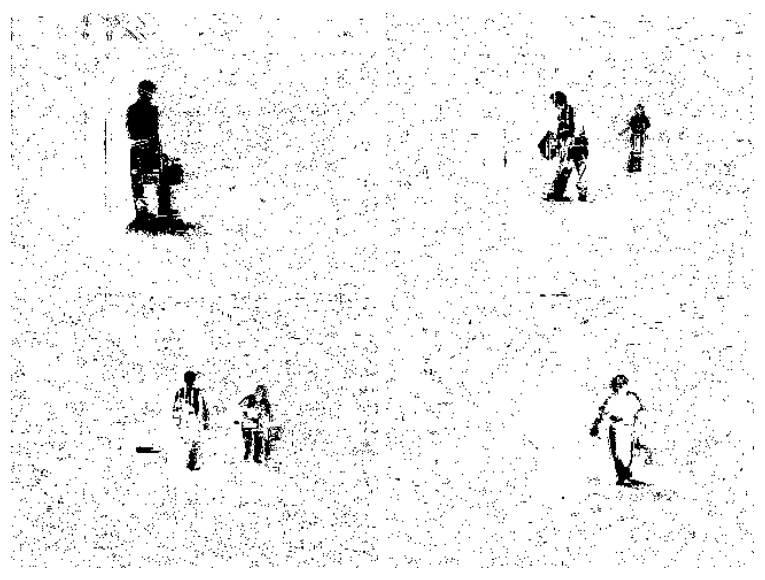

Fig. 5. $\Sigma \Delta+$ Zipf

quantitative) performances for difficult sequences with radial movement and non-rigid object. As its complexity remains low this algorithm is well suited for very light embedded systems. Future work will consider other difficult sequences with the presence of clutter like snow, rain or moving trees.

\section{REFERENCES}

[1] L. Lacassagne; A. Manzanera; J. Denoulet; A. Mérigot, "High performance motion detection: Some trends toward new embedded architectures for vision systems," JRTIP, october 2008

[2] M. Piccardi, "Background subtraction techniques: a review," in Conference on Systems, Man and Cybernetics. IEEE, 2004, vol. 4, pp. 30993104.

[3] J. Richefeu A. Manzanera, "robust and computationally efficient motion detection algorithm based on sigma-delta background estimation," in ICVGIP. IEEE, 2004.

[4] L. Lacassagne J. Denoulet, G. Mostafaoui and A. Mérigot, "Implementing motion markov detection on general purpose processor and associative mesh," in CAMP. IEEE, 2005.

[5] A. Manzanera, "Sigma-delta background subtraction and the zipf law," in CIARP. LNCS, 2007, vol. 28-2, pp. 42-51.

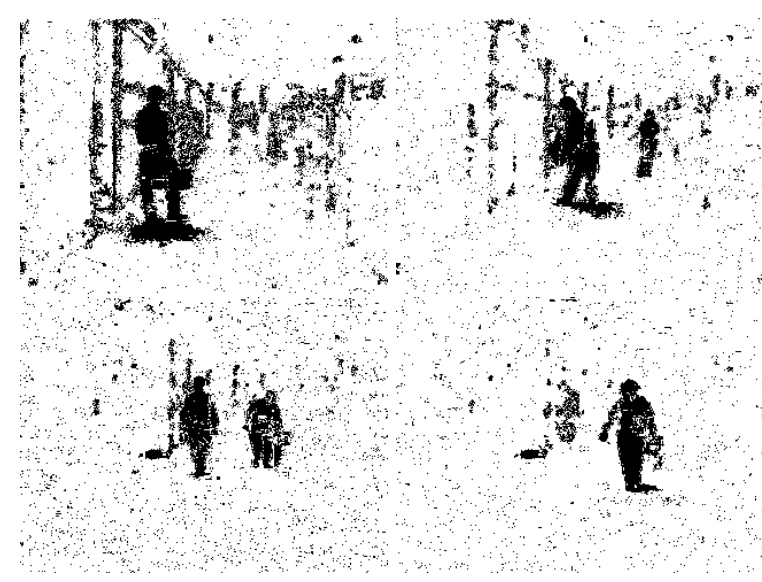

Fig. 6. conditional $\Sigma \Delta$

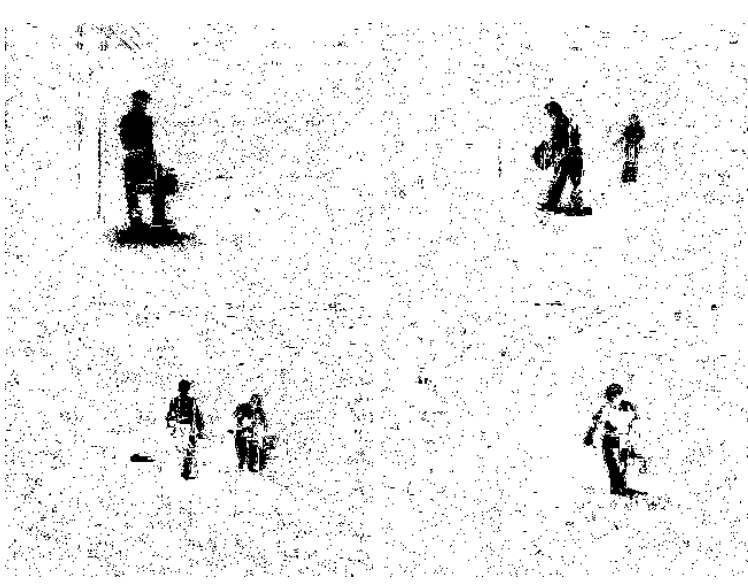

Fig. 7. Hierarchical $\Sigma \Delta$
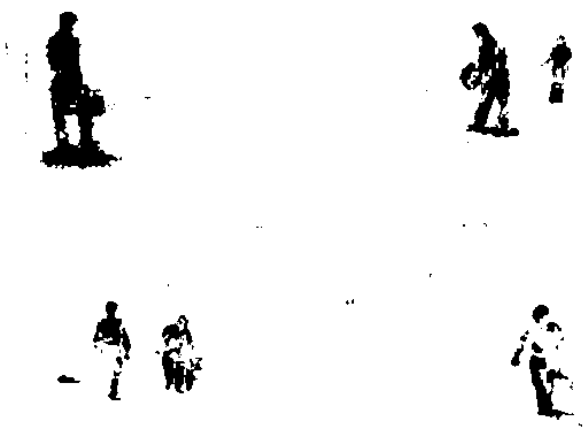

Fig. 8. Hierarchical $\Sigma \Delta+$ Morpho 